\title{
Inferring the dust properties and density distribution in the outer envelope of IRC +10216 from scattered Galactic light
}

\author{
T. Lunttila and M. Juvela
}

\author{
Observatory, University of Helsinki, PO Box 14, 00014 University of Helsinki, Finland \\ e-mail: tlunttil@astro.helsinki.fi
}

Received 5 March 2007 / Accepted 13 April 2007

\begin{abstract}
Aims. We determine the dominant dust grain size and dust density distribution in the outer envelope of the high mass-loss carbon star IRC +10216 and estimate the interstellar radiation field (ISRF) intensity and colours near IRC +10 216.

Methods. We use Monte Carlo radiative transfer simulations to calculate the surface brightness distribution of the envelope due to scattered Galactic light. Calculations are made with several dust models and cloud density structures using realistic anisotropic interstellar radiation field. The results of the calculations are compared with measurements reported in the literature.

Results. The shape of the brightness profile of the cloud at different optical wavelengths implies that the dominant dust grain radius in the outer envelope of IRC +10216 is at least $0.1 \mu \mathrm{m}$. The shape of the brightness profiles at large offsets shows that the cloud density structure is steeper than $r^{-2}$, indicating that the mass-loss rate has increased with time. The peak brightness and colours of the nebula cannot be reproduced simultaneously with the observed cloud shape with any of the dust models tested, if commonly used values for interstellar radiation field intensity are adopted. The best fit to the data is obtained with large grains (grain radius $a>0.25 \mu \mathrm{m}$ ); our simulations then imply a $\sim 30 \%$ lower interstellar radiation field intensity in $B$ band than given by Galaxy models. However, it is possible that detailed modelling including, e.g., porous grains and realistic dust size distributions could eliminate the discrepancy.
\end{abstract}

Key words. stars: carbon - stars: individual: IRC +10216 - stars: circumstellar matter - ISM: dust, extinction - scattering

\section{Introduction}

Intermediate-mass stars lose a large fraction of their initial mass during their asymptotic giant branch (AGB) phase. The lost mass forms an envelope around the star, sometimes completely absorbing the stellar light at visible and shorter wavelengths. IRC $+10216\left(l=221.4^{\circ}, b=45.0^{\circ}\right)$, a nearby AGB carbon star, is a prime example of AGB stars with copious mass loss. Its circumstellar envelope absorbs nearly all light at short wavelengths and re-emits the energy as infrared radiation. Because of its proximity IRC +10216 has been observed extensively, and it is an important prototype for studying mass loss in AGB stars. Furthermore, AGB carbon stars are believed to be the main source of carbonaceous dust in the interstellar matter, and observations of its envelope can also be used to improve models of newly formed interstellar dust.

Most studies of IRC +10216 have been done in the infrared and millimetre domains, where both the stellar scattered light and the thermal emission from the heated dust can be detected in the inner envelope. Early $V$ band photographs of the object showed a small, faint nebulosity that was interpreted as scattered stellar light. However, deep CCD-images at visual and ultraviolet wavelengths obtained by Crabtree et al. (1987) and more recently by Mauron \& Huggins (1999), Mauron et al. (2003, hereafter MLL03), and Leão et al. (2006) show an extended envelope reaching out to at least $200^{\prime \prime}$ from the star. In the $B$ band and at shorter wavelengths the surface brightness does not reach the maximum at the centre, but some $10-15^{\prime \prime}$ outwards (referred to as the plateau in MLL03). The "dark core-bright rim" surface brightness profile is a clear indication that the observed surface brightness is mostly due to external radiation scattered by strongly forward scattering particles rather than due to the light from the central star. At first sight, the outer envelope of IRC +10216 appears spherically symmetric, but deep images show the existence of several incomplete arcs spanning $30^{\circ}$ to $90^{\circ}$. Detailed images of the inner envelope reveal polar outflow cavities, but also more complex structures (Tuthill et al. 2000).

IRC +10216 is one of the brightest infrared sources outside the Solar System. A number of studies have focused on modelling the spectral energy distribution (e.g. Bagnulo et al. 1995; Skinner et al. 1998), and also on IR interferometric visibility curves (e.g. Groenewegen 1997; Men'shchikov et al. 2001, hereafter Men01). Regarding the properties of the dust, the results from different studies are conflicting. Small (radius $0.05-0.10 \mu \mathrm{m})$ dust grains have been suggested in Martin \& Rogers (1987, hereafter MR87) and Bagnulo et al. (1995), while in Skinner et al. (1998) a size distribution with grains as large as $0.4 \mu \mathrm{m}$ is favoured. Similar discrepancies exist in dust mass-loss rates with different studies suggesting values from $2.1 \times 10^{-8}$ (Groenewegen et al. 1998) to $2.3 \times 10^{-7} M_{\odot} \mathrm{yr}^{-1}$ (Skinner et al. 1999).

Another method for obtaining information about the dust grains and cloud structure is by means of modelling the surface brightness of the envelope caused by scattered interstellar radiation field (ISRF). Although observations of scattered Galactic light have been used in determining the dust properties in dark nebulae since the 1930s (Struve \& Elvey 1936; Struve \& Story 1936), and since the 1970s using computer simulations (Mattila 1970; Witt \& Stephens 1974), few works have applied this method to IRC +10216 . Compared to modelling the dust emission and scattered light from the central star, the study of the scattered ISRF has some clear advantages. The geometry is 
much simpler; the inner structure of the envelope is known to be highly complex, whereas the outer envelope is more or less spherical. Furthermore, simulations of an externally illuminated cloud are in some sense more robust than those of a cloud illuminated by an embedded star. Because the average density of the cloud decreases steeply when moving outwards, density inhomogeneities in the outer envelope are likely to be optically thin, and they have mostly local effects on the surface brightness. Moreover, when the radiation is impinging on the cloud from all directions, a small dense clump does not have a pronounced dark shadow. In contrast, an optically thick clump near the star can significantly change the stellar radiation reaching the outer parts of the cloud. Studying scattered Galactic light is also useful in that it gives information complementary to what is gained from observing dust emission and scattered stellar light. While the latter methods are best suited for studying the dust near the star, scattered Galactic light gives information on the outer part of the envelope.

Besides determining dust properties and cloud structure, the study of scattered Galactic light has another potentially important application. The intensity of the ISRF is of great importance in many fields of Galactic astronomy. Direct measurements can be used to probe the ISRF only in the solar neighbourhood. To calculate the ISRF elsewhere in the Galaxy, models of the distribution of stars and dust have been constructed (e.g. Mattila 1980; Mathis et al. 1983; Porter \& Strong 2005). Analysis of the Galactic light scattered by a dust cloud can be used to estimate the ISRF near the cloud, thereby providing a method for validating the results of the Galaxy models. While IRC +10216 is relatively nearby, $110-135 \mathrm{pc}$ according to recent estimates (Groenewegen et al. 1998), its high Galactic latitude $b \approx+45^{\circ}$ places it above the Galactic plane at a height of $z \approx 100 \mathrm{pc}$. Consequently, it can be used to study the change of the ISRF intensity with the distance from the Galactic plane.

This paper follows the works of MR87, Crabtree \& Rogers (1993) and MLL03. The halo seen around the star was first interpreted as scattered Galactic light by MR87. They also calculated the brightness profile of the envelope based on their dust and cloud models. The same approach was used in MLL03, except that they used cloud and dust models proposed in Groenewegen (1997) and compared the results to their own observations. In both MR87 and MLL03 considerable differences between the calculated profiles and the observations were found. One possible explanation is that in both these articles the ISRF illuminating the cloud was assumed to be isotropic. However, it is known that near the Sun the interstellar radiation intensity towards low Galactic latitudes is 5-10 times as intense as towards the Galactic poles, and for a vantage point above the Galactic plane the effect is likely to be even stronger (Mattila 1980). There is also some variation with Galactic longitude. As the dust particles in the envelope are believed to be strongly forward scattering, the illumination coming from behind the cloud is especially important.

The aim of this paper is to present a more detailed study of scattering in the envelope of IRC +10216 . In particular, we examine the effect of a realistic, anisotropic ISRF. Also, whereas MR87 and MLL03 used spherically symmetric cloud models, our fully 3D-model allows us to study the effect of distinct partial shells of higher density. Furthermore, we explore how the modification of the dust properties changes the surface brightness profiles. We use several physical dust models suggested for the object in previous studies and also some dust models defined only by their scattering properties. The results of our modelling are compared with the observations presented in MLL03.

\section{Radiative transfer modelling}

We use the Monte Carlo radiative transfer code described in Juvela \& Padoan (2003) and Juvela (2005) to model the surface brightness distributions. The code allows the use of both spherically symmetric and fully three-dimensional models. Hundreds of simulation runs were made with different parameters. A single run consists of the simulation of 10-200 million photon packages, which takes from $10 \mathrm{~min}$ to a few hours on a $3.0 \mathrm{GHz}$ Pentium 4.

\subsection{Cloud structure}

So far most modelling of IRC +10216 has been done with a spherically symmetric cloud with density $\rho \propto r^{-2}$. This corresponds to a stable outflow of matter from the star with constant velocity. The outflow velocity $v$ is well constrained by observations (e.g. Olofsson et al. 1982; Knapp \& Morris 1985). In our simulations we take $v=15 \mathrm{~km} \mathrm{~s}^{-1}$, which corresponds to a cloud expansion of $24^{\prime \prime}$ in 1000 years at our assumed distance of $130 \mathrm{pc}$. The uncertainty in the dust mass-loss rate $\dot{M}_{\mathrm{d}}(t)$ is much greater with values given in various works differing by a factor of ten. The values have been obtained with different methods that may probe the density in different parts of the cloud, and the differences could be at least partly explained by a changing mass-loss rate.

With constant outflow velocity $v$, and mass-loss history $\dot{M}_{\mathrm{d}}(t)$, the resulting cloud density structure is obtained from the continuity equation

$4 \pi v r^{2} \rho(r)=\dot{M}_{\mathrm{d}}\left(-\frac{r}{v}\right)$,

where we have chosen the convention that $t=0$ corresponds to the present and $t<0$ to the past. We explore the effect of changing $\dot{M}_{\mathrm{d}}$ by using a family of exponentially changing massloss rates. We model the dust mass-loss as $\dot{M}_{\mathrm{d}}(t)=\dot{M}_{\mathrm{d}}(0) \mathrm{e}^{\alpha t}$ with several different values for the rate constant $\alpha$ and current mass-loss rate $\dot{M}_{\mathrm{d}}(0)$. From Eq. (1), the dust density at distance $r$ is then

$\rho(r)=\frac{\dot{M}_{\mathrm{d}}(0) \mathrm{e}^{-\alpha r / v}}{4 \pi v r^{2}}$

The rate constants $\alpha$ considered here are between $-2.0 \times$ $10^{-4}$ and $+5.0 \times 10^{-4} \mathrm{yr}^{-1}$, while the current dust mass-loss rates $\dot{M}_{\mathrm{d}}(0)$ range from $2.5 \times 10^{-8}$ to $8.0 \times 10^{-7} M_{\odot} \mathrm{yr}^{-1}$.

For the radiative transfer calculations, the spherically symmetric clouds are discretised into 100 shells with the thickness of the shells increasing outwards. The outer edge of the cloud is set at the distance of $50000 \mathrm{AU}\left(\sim 385^{\prime \prime}\right)$ from the star. While in MLL03 the light scattered by the envelope was detected out to $\sim 200^{\prime \prime}$ from the star, IRAS observations show that the true extent of the cloud is at least $9.5^{\prime}$ (Young et al. 1993). However, provided that the assumed outer limit is large enough $\left(\gtrsim 200^{\prime \prime}\right)$, it has little effect on the surface brightness of the innermost $\lesssim 100^{\prime \prime}$ region.

In addition to smooth spherically symmetric models, we have performed some calculations for clouds with a shell structure similar to that seen in recent deep images (Leão et al. 2006). Even though we cannot exactly replicate the observed shell structure, we can study the general effect of distinct shells. Instead of placing the shells exactly at observed locations, we use randomly placed shells added to a $r^{-2}$-cloud. We employ the shell properties obtained in Mauron \& Huggins (2000) and 
Leão et al. (2006): thickness 1-2" (130-260 AU) ${ }^{1}$, density contrast between the shells and the intershell regions $2-4$, and shell opening angle $30-90^{\circ}$. We also do some simulations with higher shell-intershell density contrast. Some of the partial shells do not produce a visible arc, e.g. those on the far side of the cloud, and instead of 5-20" distances between the visible arcs we use 2-8" (260-1040 AU) distances between the shells. The threedimensional cloud is discretised as a $511^{3}$ grid with cubical cells. To achieve good enough resolution to describe the shell structure while keeping the memory consumption of the simulations manageable, the cloud outer radius was reduced to $30000 \mathrm{AU}$ $\left(\sim 230^{\prime \prime}\right)$.

Some models of IRC +10216 , e.g. Men01 and Skinner et al. (1998), include details of the complex inner envelope, in particular the bipolar cavities. While the inner structure of the cloud is very important when studying the infrared emission from the dust heated by the central star, it has very little effect on the distribution of scattered Galactic light. Therefore, we do not model the innermost $100 \mathrm{AU}$ of the envelope in detail. The volume of the core is less than one millionth of the total cloud volume used in simulations, and in a typical model the $B$ band optical thickness from $r=100 \mathrm{AU}$ to the outer edge of the cloud is $\sim 10$. Thus, very few Galactic ISRF photons reach the cloud centre, and scatter back to the observer.

\subsection{Dust models}

There is a consensus among researchers that more than $80 \%$ of solid material in the envelope of carbon stars is amorphous carbon, and a broad emission feature at $\sim 11 \mu \mathrm{m}$ indicates that also silicon carbide must be present (Wallerstein \& Knapp 1998, and references therein). There are possibly also some sulphides (Men01). However, as noted in the introduction, the grain size distribution in the envelope of IRC +10216 is very uncertain. We focus on size distributions with a single dominant grain size. A wide range of sizes from very small (grain radius $a=0.01 \mu \mathrm{m}$ ) to very large $(a=1.0 \mu \mathrm{m})$ is used. In all our models we assume that both the carbon and $\mathrm{SiC}$ grains have the same size. In some works, e.g. Men01, the relative abundances and size distributions of dust components are allowed to change throughout the cloud. While this effect is important near the star, where different components condense at different distances, the conditions in the outer envelope are much more stable. Indeed, in Men01, where a very detailed modelling of the dust envelope of IRC +10216 was performed, a single dust mixture outside the radius of $100 \mathrm{AU}$ is recommended. Because we are only interested in the Galactic light scattered in the outer envelope a single dust mixture is sufficient.

We assume that the dust grains are homogeneous spheres, so that their absorption and scattering cross sections and the scattering phase function can be calculated using Mie theory. We ignore the effect of sulphides, and concentrate only on grains consisting of amorphous carbon and silicon carbide. Different studies on IRC +10216 suggest $\mathrm{SiC}$ abundances ranging from less than 5 to about $15 \%$. In our simulations we use a dust mixture with $90 \%$ of the grains consisting of amorphous carbon, and $10 \%$ of $\mathrm{SiC}$ ( $\sim 15 \%$ by mass). We use optical properties for amorphous carbon as given by Rouleau \& Martin (1991, type AC1), and for $\mathrm{SiC}$ we take the values recommended by Pegourie (1988). We adopt material densities $\rho_{\mathrm{AC}}=2.0 \mathrm{~g} \mathrm{~cm}^{-3}$ for amorphous carbon, and $\rho_{\mathrm{SiC}}=3.16 \mathrm{~g} \mathrm{~cm}^{-3}$ for silicon carbide, yielding the average density of $\bar{\rho}=0.1 \rho_{\mathrm{SiC}}+0.9 \rho_{\mathrm{AC}}=2.12 \mathrm{~g} \mathrm{~cm}^{-3}$. With

\footnotetext{
1 We use a uniform distribution between the given limits.
}

the material properties adopted, the simulation results are rather insensitive to small changes in the abundance of $\mathrm{SiC}$ in the envelope.

Besides physical dust models, we do some calculations with dust grains defined by their single scattering albedo $\omega$, and asymmetry parameter $g$. For these models, we use the Henyey-Greenstein phase function (Henyey \& Greenstein 1941):

$\Phi(\theta)=\frac{1}{4 \pi} \frac{1-g^{2}}{\left(1+g^{2}-2 g \cos (\theta)\right)^{3 / 2}}$,

where $\theta$ is the scattering angle.

\subsection{Incident radiation field}

The flexibility of Monte Carlo methods for solving the radiative transfer equation makes it easy to use arbitrary distributions of radiation impinging on the cloud. Because the scattering phase functions of most dust models proposed for the dust ejected from a carbon star are highly forward scattering, especially the radiation field incident on the far side of the cloud with respect to the Earth should be modelled carefully. Therefore, instead of isotropic external radiation field used in earlier studies of IRC +10216 , we use a realistic distribution based on observations and modellings. We focus mostly on simulations of surface brightness at $440 \mathrm{~nm}$ ( $B$ band), but some simulations are done also at $365(U)$ and $550 \mathrm{~nm}(V)$. The simulations are done only at the effective wavelengths for the filter pass bands, as neither the ISRF nor the dust scattering properties change very rapidly at these wavelengths.

Our model for the ISRF directional distribution uses the measurements made by the Pioneer 10 space probe as reported by Toller (1981), which are unique in that they are completely free of any contamination by zodiacal light and atmospheric disturbances. Because the measurements give the ISRF only for an observer near the Galactic plane, they have to be transformed to the location of IRC +10216 at $z \approx 100 \mathrm{pc}$. We use the method from Witt et al. (1990):

$$
\operatorname{ISRF}\left(z=100 \mathrm{pc}, b, l, \lambda_{1}\right)=P_{10}\left(b, l, \lambda_{2}\right) \frac{M\left(z=100 \mathrm{pc}, b, \lambda_{1}\right)}{M\left(z=0 \mathrm{pc}, b, \lambda_{2}\right)},
$$

where $P_{10}$ is the ISRF intensity at Galactic latitude $b$, longitude $l$ and wavelength $\lambda_{2}$ as measured by Pioneer 10 , and $M(z, b, \lambda)$ is the ISRF intensity given in Mattila (1980, Tables V and VII) at the specified distance above the Galactic plane, Galactic latitude, and wavelength. In Mattila's tables the ISRF intensity is given for 11 latitudes, between which we use linear interpolation. We apply the Pioneer 10 blue band $\left(\lambda_{\text {eff }}=441 \mathrm{~nm}\right)$ measurements in constructing the ISRF distribution also in $U$ and $V$ bands, because no measurements were made at those wavelengths. While we consider only one ISRF angular distribution for each passband, the $U, B$, and $V$ band mean ISRF intensities, $J_{v}^{\mathrm{ISRF}}(\lambda)=\frac{1}{4 \pi} \int_{4 \pi} I_{v}^{\mathrm{ISRF}}(\lambda) \mathrm{d} \Omega$, are free parameters that we try to determine.

Pioneer 10 data are missing for $\mathrm{a} \sim 0.65$ sr region which is the area close to the Sun as seen from the probe. Unfortunately, this missing section is around $b=+25^{\circ}$ to $+50^{\circ}, l=200^{\circ}$ to $230^{\circ}$, i.e. in the direction of IRC +10216 . We have interpolated the Pioneer 10 measurements for the missing region. Because of the smoothness of the surface brightness at these relatively high Galactic latitudes, the interpolation is not expected to introduce large uncertainties. Other, mainly ground-based measurements show that this is a reasonable assumption; there are no highly 
distinct features in the ISRF towards IRC +10216 (for a review of ISRF measurements, see Leinert et al. 1998, Chapter 11). One can also estimate the magnitude of possible errors by calculating the surface brightness when the cloud is illuminated only by the patch of sky with interpolated intensities; the result gives the fraction of surface brightness caused by radiation coming from that part of the sky. Simulations show that this fraction is usually less than $25 \%$, but with very strongly forward scattering grains (asymmetry parameter $g \approx 0.9$ ) it can reach $60 \%$. Therefore, modest ( $\$ 15 \%)$ errors in the ISRF intensity towards the missing region possibly caused by the interpolation are not a major source of error, unless the scattering phase function is very strongly forward throwing.

The anisotropy of the constructed ISRF is significant. The lowest intensity is seen towards the region around the Galactic north pole. In the $B$ band the ISRF intensity towards the Galactic south pole is higher by a factor of $\sim 2.2$. Towards low Galactic latitudes $\left(|b|<5^{\circ}\right)$ the ISRF is, on average, approximately 20 times and towards the region of highest intensity at $b \approx 0^{\circ}, l \approx 0^{\circ}$ more than 50 times as intense as towards the Galactic north pole. The anisotropy of the ISRF in the $U$ and $V$ bands is similar.

We have ignored the contribution of the central star embedded in the cloud in all our simulation runs. This is justified because in the $U$ and $B$ bands the stellar light is almost completely absorbed by the inner layers of the envelope, and at these wavelengths the dust emission is negligible. Including the radiation from the star in our simulations would only mean longer calculations without much visible effect. In the $V$ band the scattered light from the star dominates the surface brightness for small offsets, but it diminishes rapidly with increasing distance. We have not included the light from the central star in our $V$ band simulations.

We do not include the radiation transmitted through the cloud without scattering in the results of our simulations. The location of IRC +10216 at high Galactic latitude in a region of the sky where the interstellar extinction is small means that there is little diffuse background from behind it. The radiation incident on the far side of cloud is mainly from individual stars. Bright stars seen through the envelope were masked out before calculating the brightness profiles presented in MLL03.

\section{Modelling results and interpretation}

The problem of determining the properties of the dust grains, the density distribution of the envelope, and the intensity of the incident ISRF from observations of scattered light is inherently ill-posed; the solution is very sensitive to measurement noise, and it may not be unique. In the present case we consider the data from three wavelengths, where the surface brightness of the cloud has been measured by MLL03 with deep images to large offsets. However, even within our limited model space of six parameters - one parameter for the grain size, two parameters describing the density structure of the envelope, and the average ISRF intensities in the $U, B$, and $V$ bands - it is possible to find many models that fit the observations very well if the parameters are allowed to change freely. Nevertheless, the results allow us to set some rather tight limits on the parameters.

In this section we present the results of our simulations described above, and reanalyse the observations of MLL03. We first examine the brightness profile shape, and use the results to determine the density structure of the cloud and to set some limits on the grain size. Thereafter, we study the absolute surface brightness of the envelope in order to improve the constraints on

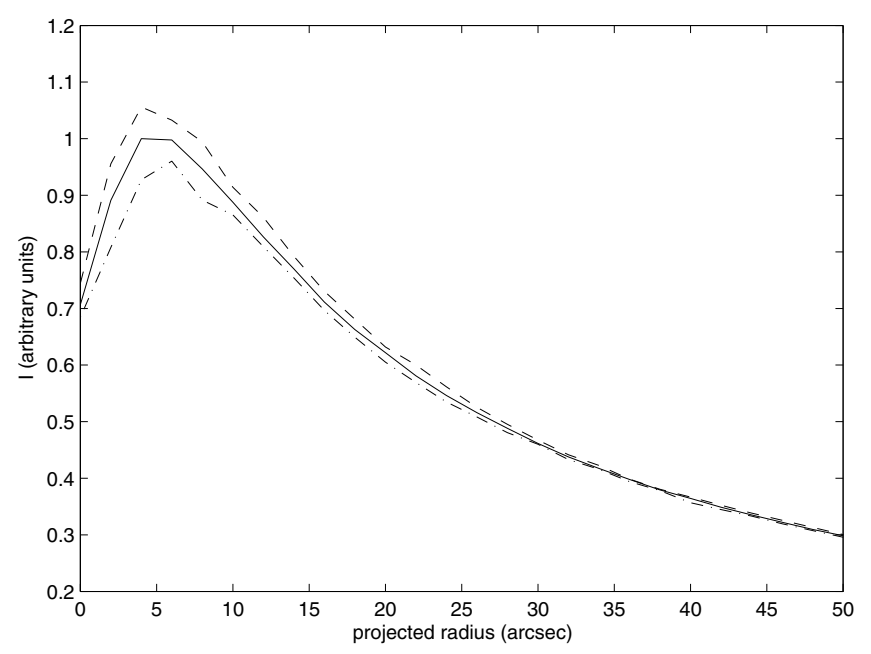

Fig. 1. Simulated brightness profiles of IRC +10216 in $B$ band with parameters $a=0.20 \mu \mathrm{m}, \dot{M}_{\mathrm{d}}(0)=5 \times 10^{-8} M_{\odot} \mathrm{yr}^{-1}$, and $\alpha=0 \mathrm{yr}^{-1}$. Solid line: azimuthally averaged profile, dashed line: profile towards the Galactic plane, dash-dotted line: profile away from the Galactic plane.

the grain size, and to gain information on the ISRF intensity and colours. Finally, we examine the effect of distinct dense shells on the global surface brightness distribution.

\subsection{Shape of the brightness profiles}

Figure 1 shows examples of the simulated surface brightness profiles of the envelope. Because of the anisotropic ISRF, the profiles towards different directions are not identical, although the cloud is spherically symmetric. However, the brightness asymmetry is quite small, and it is limited to the inner part of the cloud. In MLL03 it is reported that $U$ band images show that the cloud may indeed be slightly brighter on its south-west side that is facing the Galactic plane, but deeper images are needed to study the asymmetry. Because it is not possible to extract quantitative information on the degree of the ISRF anisotropy from the available surface brightness maps of the envelope, the following analysis focuses on the azimuthally averaged brightness profiles. The observed profiles shown in Fig. 2. of MLL03 are averaged over 4 " annuli, and the same averaging was performed to model profiles computed from the simulated surface brightness maps.

We studied in most detail the $B$ band brightness profiles presented in Fig. 2 of MLL03; the $U$ band profiles are noisy due to the very low surface brightness of the cloud in that bandpass, while at least the inner parts of the $V$ band profiles are severely contaminated by the light from the central star. Based on the differences between the two measured profiles (i.e. from the Canada-France-Hawaii Telescope (CFHT) and the Observatoire de Haute Provence (OHP) $1.2 \mathrm{~m}$ telescope), it is evident that also the $B$ band profiles have considerable uncertainties at least at offsets $\gtrsim 50^{\prime \prime}$. The parts inwards and outwards of the brightness peak are studied separately. The reason is that the brightness of the outer envelope is best suited for the study of the density distribution; when the line-of-sight optical thickness $\tau$ is $\lesssim 1$, the observed brightness conveys direct information on the mass column density. In contrast, the line-of sight optical thickness through the inner parts of the cloud is large, and a major part of the observed intensity is due to backscattering. It is possible to gain information on the dust properties, in particular on the scattering phase function, but inferring the density distribution is much more difficult. 


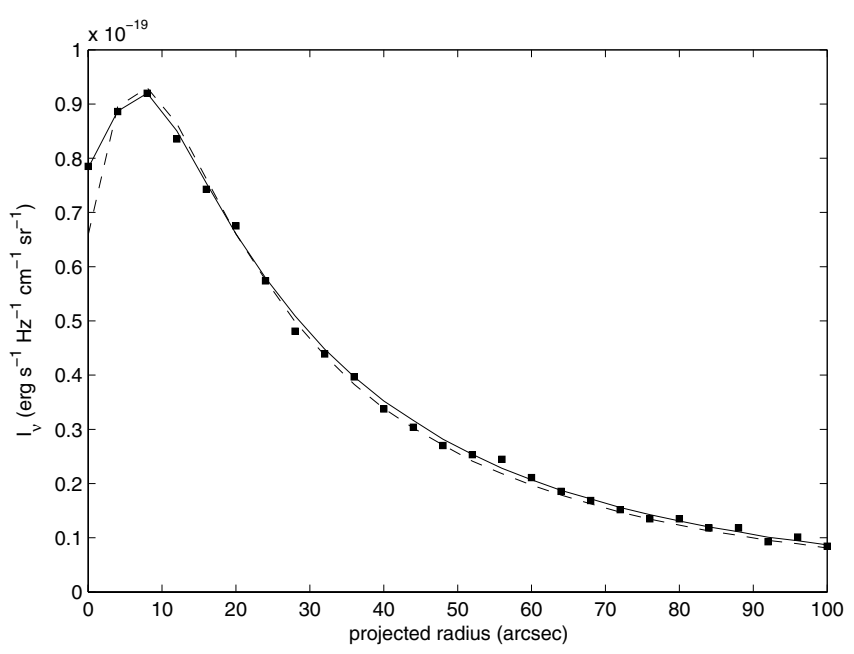

Fig. 2. The $B$ band surface brightness profile measured by MLL03 at OHP (square markers) compared with the best-fit model profiles for grain sizes $0.05 \mu \mathrm{m}$ (solid line) and $0.25 \mu \mathrm{m}$ (dashed line). The model parameters are $\dot{M}_{\mathrm{d}}=9.5 \times 10^{-8} M_{\odot} \mathrm{yr}^{-1}, \alpha=1.8 \times 10^{-4} \mathrm{yr}^{-1}$ for $a=0.05 \mu \mathrm{m}, \dot{M}_{\mathrm{d}}=8.8 \times 10^{-8} M_{\odot} \mathrm{yr}^{-1}, \alpha=1.65 \times 10^{-4} \mathrm{yr}^{-1}$ for $a=0.25 \mu \mathrm{m}$.

The density structure of the outer envelope was determined by calculating the parameter values $\alpha, \dot{M}_{\mathrm{d}}(0)$ and the mean $B$ band intensity $J_{v}^{\mathrm{ISRF}}(\lambda=441 \mathrm{~nm})$ that provide the best fit to the observed surface brightness profiles for offsets $12^{\prime \prime} \leq \theta \leq 120^{\prime \prime}$. We minimised the residual

$\chi^{2}\left(J_{v}^{\mathrm{ISRF}}, \alpha, \dot{M}_{\mathrm{d}}(0)\right)=\sum_{k=3}^{30}\left[I_{\mathrm{o}}\left(\theta_{k}\right)-I_{\mathrm{m}}\left(\theta_{k} ; J_{v}^{\mathrm{ISRF}}, \alpha, \dot{M}_{\mathrm{d}}(0)\right)\right]^{2}$,

where $I_{\mathrm{o}}$ is the observed and $I_{\mathrm{m}}$ the modelled profile, and $\theta_{k}=$ $4 k^{\prime \prime}$ are the observed offsets. Parameters $\dot{M}_{\mathrm{d}}(0)$ and $\alpha$ were allowed to vary freely over a wide range, but the mean ISRF intensity $J_{v}^{\mathrm{ISRF}}$ was allowed to vary only little, so that the predicted peak brightness was between 0.95 and 1.05 times the observed maximum intensity. This was done to ensure that the most distinctive feature of the brightness profile was correctly fitted. The fitting was done separately to both the CFHT and OHP data that were presented in MLL03.

Figure 2 shows two of the brightness profiles obtained with the optimal parameters for different dust models. For offsets $\gtrsim 12^{\prime \prime}$ the profiles are almost identical, and they match the observations very well. The results for other dust models are nearly identical. With any of our dust models, it is possible to find a cloud density structure that reproduces the observed brightness distribution of the outer part almost exactly. The bestfit value of $\alpha$ varies only little with assumed grain size, but the results for $\alpha$ from OHP and CHFT profiles differ by a factor of 2.2: from the wider OHP profile we obtain $\alpha=(1.85 \pm 0.1) \times$ $10^{-4} \mathrm{yr}^{-1}$, while from the CFHT data we get $\alpha=(4.0 \pm 0.2) \times$ $10^{-4} \mathrm{yr}^{-1}$. On the other hand, the estimates for $\dot{M}_{\mathrm{d}}(0)$ depend heavily on the assumed dust model. The optimal $\dot{M}_{\mathrm{d}}(0)$ as a function of grain radius is depicted in Fig. 3.

Witt \& Stephens (1974) show that the depth of the dark core of a globule increases with the scattering asymmetry parameter $g$. Our simulations show the same trend, and also the increase of the core depth with the size of optically thick core relative to the cloud. The shape and width of the central depression depend heavily on the density structure of the cloud, but within the range of cloud models probed in this paper the core depth, defined as $C D=1-I_{\text {core }} / I_{\text {peak }}$, varies only a little. While the

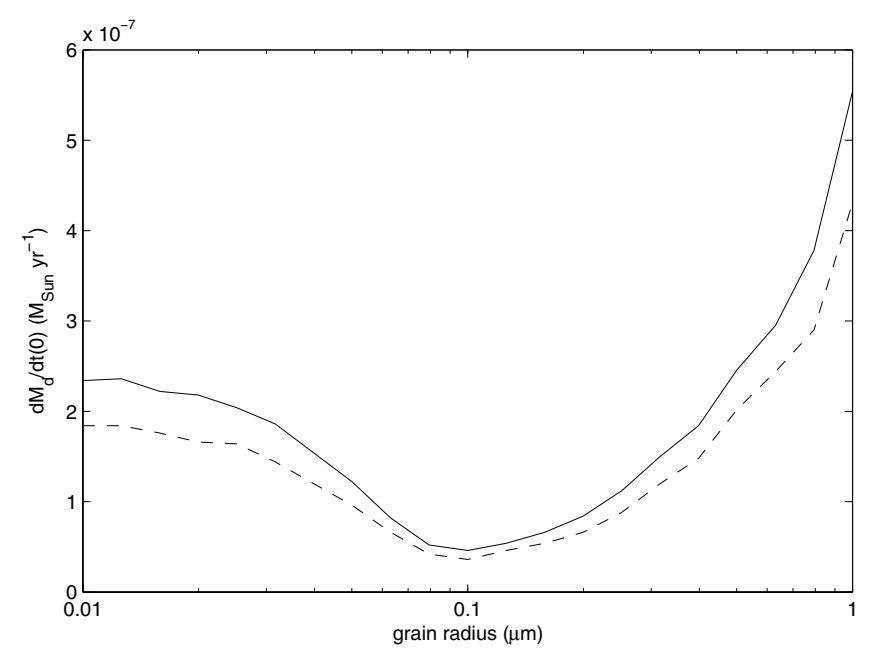

Fig. 3. Best-fit value of $\dot{M}_{\mathrm{d}}(0)$ as a function of assumed grain size. The solid line shows the values calculated from the observations of MLL03 with CFHT, the dashed line indicates the results obtained from their OHP data.

outer part of the nebula can be used to study the density structure, analysis of the brightness of the core is better suited for probing the parameters of the dust model. Unfortunately, the determination of the dust properties from the observed core depth is difficult. With very strongly peaked cloud density structures such as the ones considered in this paper, the central depression can be very steep and narrow. Our simulations show that in some cases sub-arcsecond resolution is needed to determine the actual depth of the dark core. With lower resolution the depth of the core seen in the observations is less than the actual core depth. Furthermore, in the $B$ band, where the core depth is best determined, the light from the central star is seen as a faint point-like source. Although the point source was subtracted in the analysis of MLL03 it is possible that a small part of the surface brightness of the inner envelope is caused by the diffuse scattered light from the central star. Despite these limitations, the fairly low observed core depth can be used to constrain the dust properties. Our simulations show that for all grain sizes $a \lesssim 0.12 \mu \mathrm{m}$ the core depth is consistent with the value $C D \approx 0.17 \pm 0.03$ determined by MLL03. With larger grains the dark core is deeper, with $C D \approx 0.7$ for $a=1.0 \mu \mathrm{m}$. Because of the difficulties described above we cannot set a very firm upper limit on the grain size, but very large grains with $a \gtrsim 0.4 \mu$ m predicting $C D>0.5$ seem to be excluded.

We did not analyse the $U$ and $V$ band profiles in as great a detail as the $B$ band ones because of their lower quality. Rather, we used the cloud structure inferred from the $B$ band profiles to simulate the $U$ and $V$ brightness distributions. By analysing how the simulated profiles match the observations one can constrain the dust model, and obtain additional information on the cloud structure. As with the $B$ band profiles, only offsets $12^{\prime \prime} \leq \theta \leq 120^{\prime \prime}$ are considered, and the ISRF intensity is optimised under the constraint that the peak brightness of the simulated profiles matches the observations within $5 \%$. The $V$ band profile measured at OHP cannot be used for the fitting, because the light from the central star prevents the determination of maximum scattered ISRF intensity, but in the case of the CFHT $V$ band profile the determination is possible because of the slight "knee" 


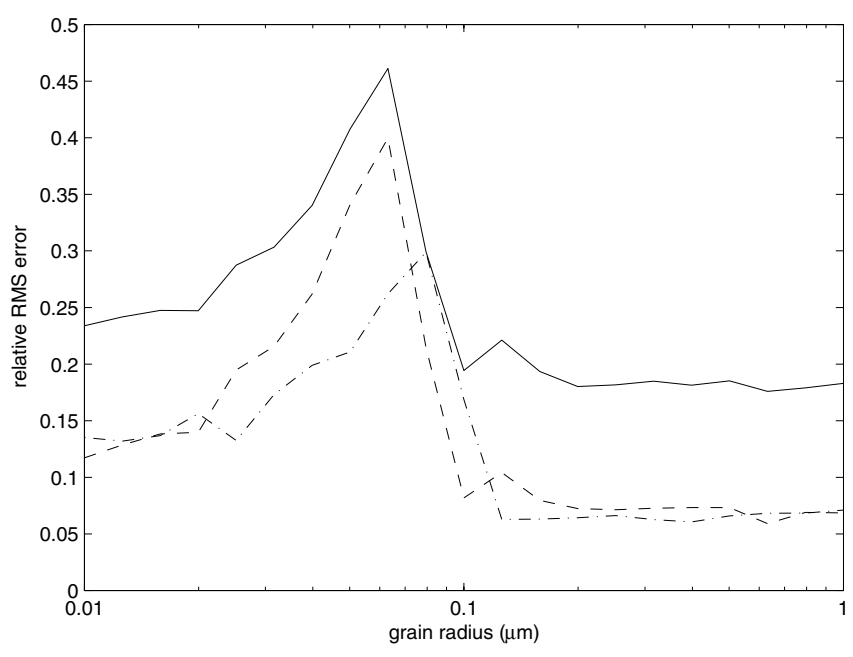

Fig. 4. Relative root-mean-square error from fitting the simulated profiles to the $U$ and $V$ band observations. Solid line: VLT $U$ band observations, dashed line: OHP $U$ band observations, dash-dotted line: CFHT $V$ band observations.

in the profile. Figure 4 displays the relative root-mean-square error

$R M S E=\sqrt{\frac{\sum_{k=3}^{30}\left[I_{\mathrm{o}}\left(\theta_{k}\right)-I_{\mathrm{m}}\left(\theta_{k}\right)\right]^{2}}{\sum_{k=3}^{30} I_{\mathrm{o}}\left(\theta_{k}\right)^{2}}}$

as a function of grain size in both $U$ and $V$ bands. The cloud structure was determined from the OHP $B$ band data; the CFHT data yield similar results. The models fit better to the OHP than the VLT $U$ band profile, mainly because the VLT profile does not show the profile well at offsets $>80^{\prime \prime}$. However, despite the differences in the measured profiles, in both cases the rms error curve has the same general form.

Comparing the simulation results to observations shows that the grain size must be large, $a>0.1 \mu \mathrm{m}$. Our calculations show that if the grain size is small, the width of the brightness profiles decreases strongly with increasing wavelength. In contrast, the observed profiles are strikingly similar in all three passbands. Figure 5 shows the observed OHP $U$ band profile with two simulated profiles. The models used in the simulations are the same as in Fig. 2. The similarity of the profile shapes at different wavelengths sets a very tight lower limit for the grain size; with grain radius $a=0.12 \mu \mathrm{m}$ the profiles show little dependence on the wavelength, but already with $a=0.10 \mu \mathrm{m}$ the shape chromatism, i.e. the change of profile shape with wavelength, is noticeable (see Fig. 4). If the grain radius is very small, $a<0.03 \mu \mathrm{m}$, the shape chromatism is not as pronounced as with slightly larger grains, but even in that case the effect should be seen in the observations.

\subsection{Absolute brightness of the envelope}

Figure 6 displays the optimal $B$ band mean ISRF intensities for different grain sizes obtained from fitting the simulated brightness profiles to the observations. Very small grains have low albedo, and therefore a relatively high ISRF intensity is required. The lowest inferred $B$ band ISRF intensity, $J_{v}^{\mathrm{ISRF}}(\lambda=440 \mathrm{~nm})=$ $2.7 \times 10^{-19} \mathrm{erg} \mathrm{s}^{-1} \mathrm{~Hz}^{-1} \mathrm{~cm}^{-2} \mathrm{sr}^{-1}$ is reached with the grain size $a \approx 0.1 \mu \mathrm{m}$. For all grain radii $a \gtrsim 0.25 \mu \mathrm{m}$ the optimal ISRF intensity is almost identical, $J_{v}^{\operatorname{ISRF}}(\lambda=440 \mathrm{~nm})=6.7 \times$ $10^{-19} \mathrm{erg} \mathrm{s}^{-1} \mathrm{~Hz}^{-1} \mathrm{~cm}^{-2} \mathrm{sr}^{-1}$. Table 1 shows the characteristics of some synthetic ISRF models. The models agree fairly well on

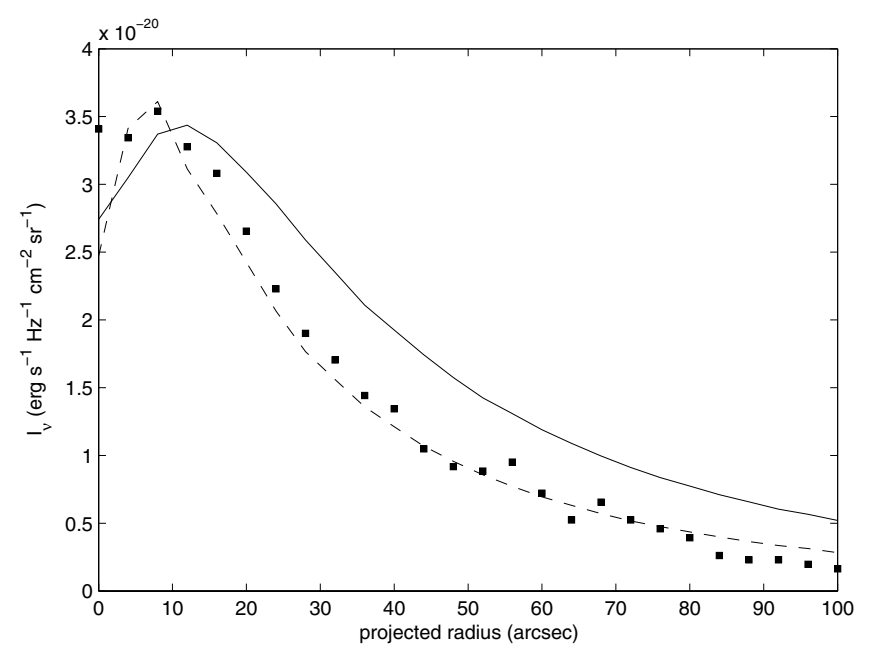

Fig. 5. The $U$ band profile measured by MLL03 at OHP (square markers) compared with two simulated profiles: grain size $0.05 \mu \mathrm{m}$ (solid line) and grain size $0.25 \mu \mathrm{m}$ (dashed line). The parameters of the cloud models are the same as in Fig. 2.

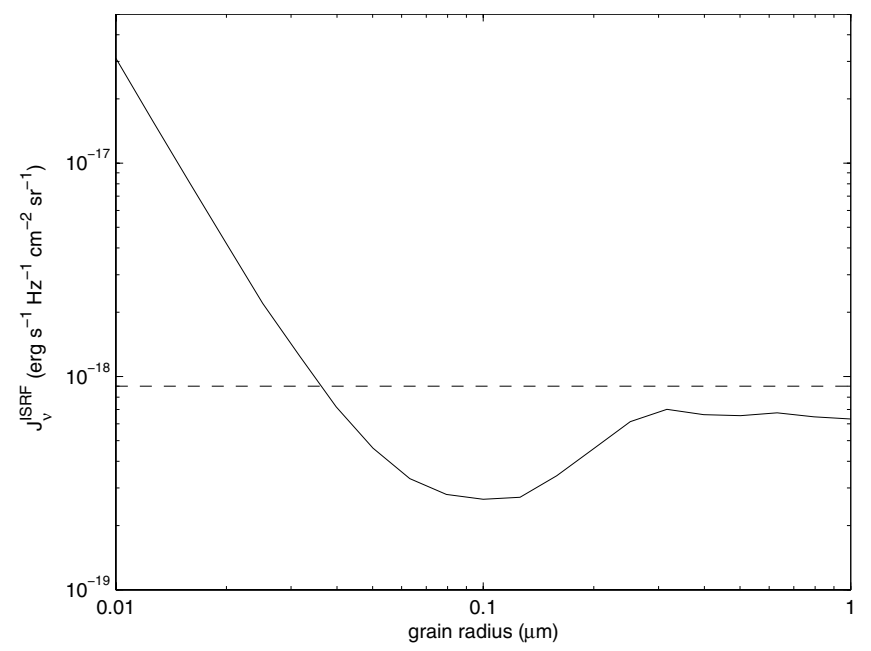

Fig. 6. The best-fit $B$ band mean ISRF intensity as a function of grain radius. The dashed horizontal line shows the approximate intensity $9.0 \times$ $10^{-19} \mathrm{erg} \mathrm{s}^{-1} \mathrm{~Hz}^{-1} \mathrm{~cm}^{-2} \mathrm{sr}^{-1}$ predicted by the Galaxy models.

the $B$ band intensity, all predicting $J_{v}^{\mathrm{ISRF}}(\lambda=440 \mathrm{~nm}) \approx(9.0 \pm$ $0.5) \times 10^{-19} \mathrm{erg} \mathrm{s}^{-1} \mathrm{~Hz}^{-1} \mathrm{~cm}^{-2} \mathrm{sr}^{-1}$. The intensity is higher than what is inferred from the surface brightness of IRC +10216 . The grain sizes $a \approx 0.1 \mu \mathrm{m}$ that can explain all aspects of the profile shape in all three passbands require an ISRF intensity lower by a factor of $\sim 3.4$. If the uncertain upper limit for the grain size set by the depth of the dark core is relaxed, the best fit to the ISRF models is reached with large grains. Nevertheless, the synthetic ISRFs still predict a field stronger by a factor of $\sim 1.35$. Only small grains with $a=0.03-0.04 \mu \mathrm{m}$ can explain the observed surface brightness, but that small a grain size would cause significant shape chromatism.

Similar discrepancy between the ISRF intensity inferred from our modelling and the predictions of the Galaxy models exists in $U$ and $V$ bands. Nevertheless, a closer examination of the colours $U-B$ and $B-V$ can yield additional insight into the grain properties and the ISRF. Figure 7 shows the $U B V$ loci of different grain sizes for two ISRF models. Mattila's synthetic ISRF gives a better fit to the observations, with grain sizes $0.06-0.12 \mu \mathrm{m}$ producing the observed colours within the errorbox. With larger 
Table 1. Characteristics of ISRF models for $R \approx 8.5 \mathrm{kpc}, z=100 \mathrm{pc}$.

\begin{tabular}{ccl}
\hline \hline $\begin{array}{l}\lambda \\
(\mu) \mathrm{m})\end{array}$ & $\begin{array}{l}J_{v}^{\text {ISRF }} \\
\left(\mathrm{erg} \mathrm{s}^{-1} \mathrm{~Hz}^{-1} \mathrm{~cm}^{-2} \mathrm{sr}^{-1}\right)\end{array}$ & Magnitudes \\
\hline \multicolumn{2}{c}{ Pioneer 10 transformed to $z=100 \mathrm{pc}^{a}$} & \\
$0.440 \quad 9.54 \times 10^{-19}$ & $B=-6.12$ \\
Mattila (1980, Table VII) & \\
$0.360 \quad 3.23 \times 10^{-19}$ & $U=-5.88$ \\
$0.440 \quad 9.03 \times 10^{-19}$ & $B=-6.06$ \\
$0.550 \quad 1.42 \times 10^{-18}$ & $V=-6.76$ \\
& & $U-B=0.18$ \\
Porter \& Strong $(2005)^{b}$ & $B-V=0.70$ \\
$0.360 \quad 5.20 \times 10^{-19}$ & $U=-6.39$ \\
$0.440 \quad 8.53 \times 10^{-19}$ & $B=-6.00$ \\
0.550 & $1.50 \times 10^{-18}$ & $V=-6.82$ \\
& & $U-B=-0.39$ \\
& $B-V=0.82$ \\
\hline
\end{tabular}

${ }^{a}$ See Sect. 2.3. ${ }^{b}$ The intensities are calculated with linear interpolation from the table available at http://galprop. stanford.edu.

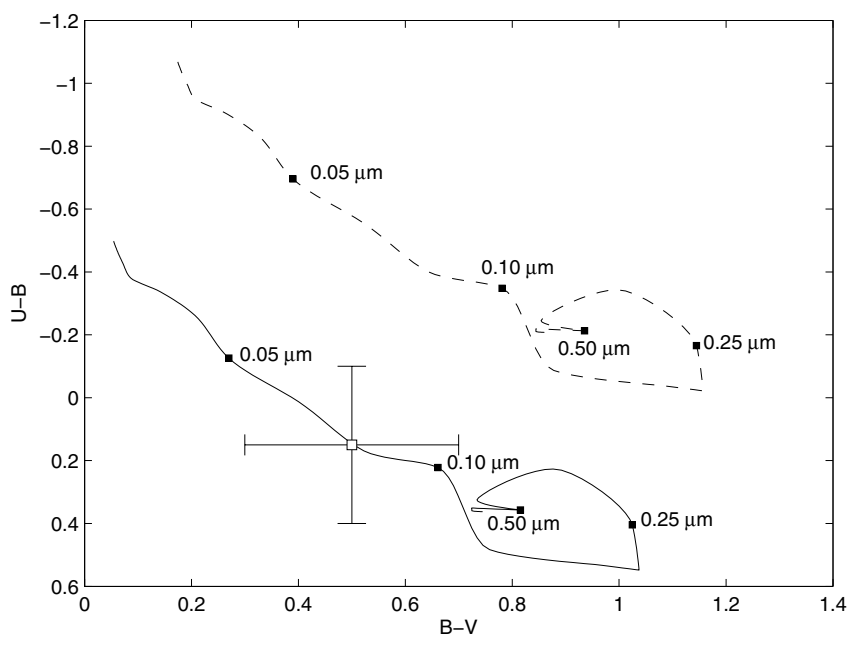

Fig. 7. Colours $B-V$ and $U-B$ with different grain sizes and two ISRF models. Solid line: Mattila (1980), dashed line: Porter \& Strong (2005) ISRF. Each line spans the range of grain sizes from $0.01 \mu \mathrm{m}$ to $1.0 \mu \mathrm{m}$. The square with errorbars indicates the colours as given in MLL03.

grains the colour is too red in $B-V$. The interstellar radiation field of Porter and Strong has $B-V$ similar to Mattila's, but much bluer $U-B$. With Porter and Strong's ISRF the dust models used cannot produce the observed $U-B$.

We also ran a series of simulations to determine what kind of scattering properties the dust grains would need to have to agree with the ISRF intensity obtained from the Galaxy models. The surface brightness was calculated using the Henyey-Greenstein scattering function and an $r^{-2}$ density distribution, i.e. we did not try to fit the full profiles to the observations. Instead, we only focus on the peak brightness of the envelope; it is almost independent of the cloud density structure. For a given dust model and ISRF intensity, the maximum brightness changes less than $10 \%$ across the range of cloud models considered in this paper.

The results from these calculations are displayed in Fig. 8 . Also shown are the albedo and asymmetry parameters in $B$ band for the physical dust models used in the simulations. The predicted peak surface brightness increases very strongly with $\omega$, but decreases with increasing $g$; when the grains scatter strongly forward, the intense radiation from the Galactic plane is not

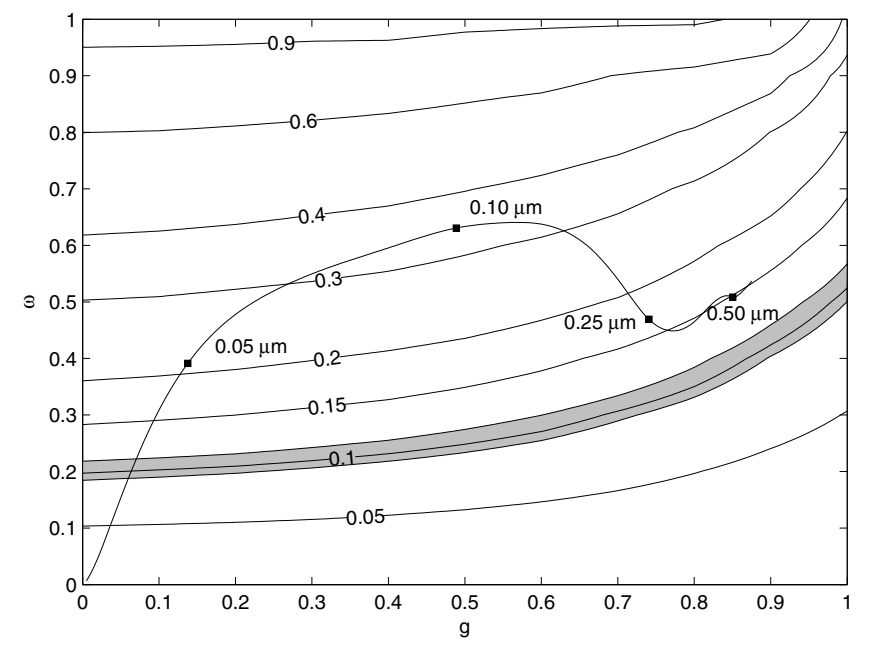

Fig. 8. Contour lines of peak surface brightness fraction of average ISRF intensity $J_{v}^{\mathrm{ISRF}}$ for different dust albedos and asymmetry parameters. The shaded region shows the range of scattering parameters required to reproduce the observed $B$ band surface brightness of $(9.2 \pm 0.7) \times 10^{-20} \mathrm{erg} \mathrm{s}^{-1} \mathrm{~Hz}^{-1} \mathrm{~cm}^{-2} \mathrm{sr}^{-1}$, assuming $J_{v}^{\mathrm{ISRF}}(\lambda=$ $440 \mathrm{~nm})=9.0 \times 10^{-19} \mathrm{erg} \mathrm{s}^{-1} \mathrm{~Hz}^{-1} \mathrm{~cm}^{-2} \mathrm{sr}^{-1}$. The line with labelled markers designates the albedo and asymmetry parameters in the $B$ band for the physical grain models with sizes $0.01-1.0 \mu \mathrm{m}$.

reflected effectively towards the Earth. There is a small, $\sim 5 \%$, difference between the peak brightness calculated using the Mie scattering function, and the Henyey-Greenstein approximation with the same values of $\omega$ and $g$, especially when $g>0.5$. With Mie scattering function the forward scattering peak is stronger, causing a higher "effective" value of $g$ and, in this case, slightly lower peak brightness.

The observed $B$ band peak brightness of $(9.2 \pm 0.7) \times$ $10^{-20} \mathrm{erg} \mathrm{s}^{-1} \mathrm{~Hz}^{-1} \mathrm{~cm}^{-2} \mathrm{sr}^{-1}$ is approximately $10 \%$ of the mean ISRF intensity predicted by the Galaxy models. It would be necessary to either reduce the $\omega$ to $\$ 0.4$ or, keeping the albedo from dust models, to increase the asymmetry parameter $g$ to $>0.9$. With the optical properties employed, it seems impossible to find a dust size distribution of spherical, homogenous grains having the required scattering properties unless the dominant grain size is small. That, however, would cause significant shape chromatism.

\subsection{Effect of distinct shells in the envelope}

Hitherto, our analysis has been limited to smooth radial density distributions. However, images have clearly shown the inhomogenous structure of the dust cocoon surrounding IRC +10216 , in particular the existence of incomplete shells of enhanced density. In Mathis et al. (2002) it is shown that if a cloud is illuminated by an embedded star, ignoring clumpy density structure may cause significant errors in the determination of dust properties. To explore whether inhomogeneities have a similar effect in the case of a cloud illuminated externally by the ISRF, we have ran simulations with some aspherical cloud models.

Figure 9 displays the azimuthally averaged surface brightness profiles of some models with distinct shells compared to the closest matching (same average mass-loss rate) $r^{-2}$ cloud. The location of the shells is the same in each case, only their density is changed. Despite the shells, the brightness profiles for density contrasts 2-4 and 4-6 are almost indistinguishable from 


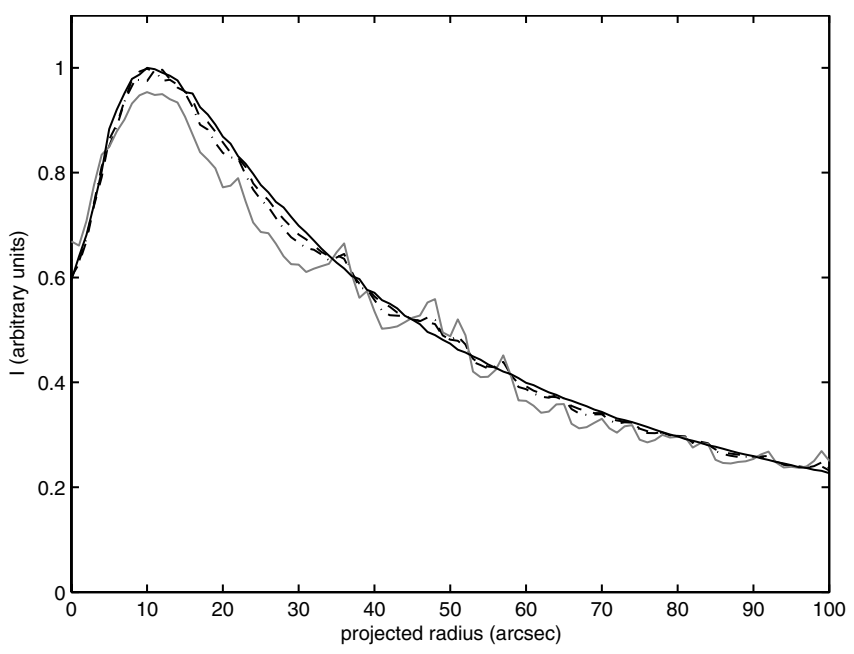

Fig. 9. Comparison of azimuthally averaged $B$ band brightness profiles for smooth $r^{-2}$-cloud (black solid line), and clouds with shell density contrast 2-4 (dashed line) 4-6 (dash-dotted line) and 10-20 (grey solid line). All clouds have the same average mass-loss rate $\dot{M}_{\mathrm{d}}=1.0 \times$ $10^{-7} M_{\odot} \mathrm{yr}^{-1}$ and grain radius of $0.25 \mu \mathrm{m}$.

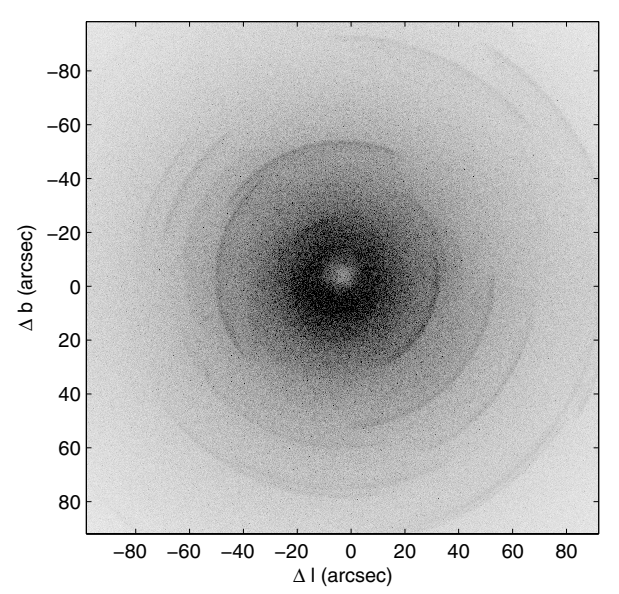

Fig. 10. Simulated image of IRC +10216 in $B$ band with distinct shells added. $\dot{M}_{\mathrm{d}}=1.0 \times 10^{-7} M_{\odot} \mathrm{yr}^{-1}$, grain radius is $0.25 \mu \mathrm{m}$, and density contrast $2-4$.

the $r^{-2}$ cloud; the azimuthal averaging smoothes out all but the strongest individual shells. Specifically, the characteristics of the profiles that were used in the analysis above - the peak brightness and the general shape of the profiles - are influenced only slightly by the presence of the shells. Even with average density contrast 15 , when approximately $40 \%$ of the cloud mass is concentrated into shells, the azimuthally averaged profile shape is very similar to the smooth $r^{-2}$ case.

The surface brightness map for the model with density contrast 2-4 is shown in Fig. 10. The bright limbs of the shells can be seen clearly, and the simulated surface brightness map resembles quite closely the actual image of IRC +10216 shown in Fig. 3 in Leão et al. (2006). With higher density contrast the shells become stronger and wider, and with contrast $\gtrsim 15$ projections of the entire shells are seen. The resolution of our simulations is not sufficient for a detailed study of the shell properties. For instance, we cannot compare the effects of thin, dense shells with those of slightly thicker, but more tenuous shells.

\section{Discussion}

The main goal of this study was to examine whether the differences between calculations and observations of scattered Galactic light in the dust envelope of IRC +10216 reported in MLL03 could be explained with more detailed modelling, in particular anisotropic ISRF. Our simulations resolve some of the discrepancies, but we are unable to find a model that could explain the observations without strongly conflicting the synthetic ISRF models. However, it is possible that a more detailed modelling of the dust properties could account for the differences.

The best constraint for the dust size distribution is set by the absence of shape chromatism. It shows that the absorption and scattering properties of the grains cannot change very much over the range of wavelengths studied. As noted already in MLL03, this points to a large grain size, $a>0.1 \mu \mathrm{m}$. The limited range of wavelengths for which photometry of the outer envelope is available hinders detailed study of the grain size distribution. In particular, it is not possible to determine an upper limit for the grain size. Observations of the scattered light from the outer envelope at longer wavelengths would be very important, because the expected onset of the narrowing of the brightness profile with wavelength is a good indicator of the dominant grain size. Although our results show that the dominant particle size must be large, a population of small particles cannot be excluded. For instance, a size distribution used in Skinner et al. (1998), with $n(a) \propto a^{-1}$ and upper size limit $a=0.4 \mu \mathrm{m}$, did not cause noticeable shape chromatism. It is also interesting to observe that the widely used dust model of Weingartner \& Draine (2001) induced very strong shape chromatism; thus, the dust in the outer envelope of IRC +10216 clearly has properties different from the general interstellar dust. The relatively low depth of the dark core of the cloud establishes an upper limit for the grain size. Although there is some uncertainty in the true core depth, our simulations show that dust grains with $a>0.4 \mu \mathrm{m}$ should produce a deeper central depression than what is observed.

Both MR87 and MLL03 found that to explain the low maximum brightness of the cloud it was necessary to reduce the incident ISRF intensity from its solar neighbourhood value by a factor of $\sim 2.5$. In contrast, Galaxy models predict that at the distance of $\sim 8.5 \mathrm{kpc}$ from the Galactic centre the ISRF should be $\sim 20 \%$ stronger at $z=100 \mathrm{pc}$ than at $z=0 \mathrm{pc}$. Our simulations confirm that for the range of grain sizes $0.05 \lesssim a \lesssim 0.2 \mu \mathrm{m}$, which includes the dust models used in MR87 and MLL03, the ISRF intensity from Galaxy models needs to be reduced by a factor of 2-3.5 to explain the observed faintness of the cloud. With grains larger than $0.25 \mu \mathrm{m}$ the discrepancy is greatly reduced. The simulations then imply an approximate upper limit of $6.6 \times$ $10^{-19} \mathrm{erg} \mathrm{s}^{-1} \mathrm{~Hz}^{-1} \mathrm{~cm}^{-2} \mathrm{sr}^{-1}$ for the $B$ band mean ISRF intensity, $\sim 30 \%$ lower than what is obtained from the Galaxy models. Very small grains with $a \approx 0.035 \mu$ m could explain the faintness of the nebula, but they are excluded because they give rise to a strong shape chromatism. Although our results concerning the peak brightness are close to those of MR87 and MLL03 when using their dust models, one cannot conclude that the ISRF anisotropy is not important; the fact that the surface brightness with the isotropic ISRF is similar to the more realistic case is merely a coincidence. For instance, with large, strongly forward scattering grains the isotropic radiation field predicts a peak brightness higher by a factor of $\sim 2$ than our anisotropic ISRF with the same mean intensity.

The difference between the ISRF intensity calculated using the Galaxy models and the results from our calculations cannot be eliminated by modifying only the grain size distribution, 
unless strong shape chromatism is accepted. However, in addition to a lower ISRF intensity near IRC +10216 there are other possible reasons for the faintness of the cloud: slightly different grain composition; different optical properties of grain materials; non-spherical, porous, or fractal-like grains; different ISRF directional distribution. In particular, studying the effects of more realistic grain shapes would be interesting. Voshchinnikov et al. (2005) have studied the effects of grain porosity on absorption and scattering properties using grain composition similar to ours. Based on their results, large $\gtrsim 0.3 \mu$ m particles with inclusions could have scattering properties that would result in an agreement between the ISRF models and our results, but a more detailed study is needed before drawing any conclusions. Also, various studies on the optical properties of amorphous carbon and silicon carbide propose slightly different refractive indices (e.g. Suh 2000; Laor \& Draine 1993), and a thorough study is needed to explore their effects. Hence, we cannot determine the $B$ band ISRF intensity near IRC +10216 with high accuracy. Still, it is clear that the intensity does not need to be as low as suggested in the earlier works MR87 and MLL03.

It is interesting to compare our results with those of Laureijs et al. (1987), who studied the high latitude dark cloud L1642 $\left(l=210.8^{\circ}, b=-36.7^{\circ}\right)$. Similar to IRC +10216 , the cloud is $\sim 100$ pc from the Galactic plane. However, its maximum surface brightness in the $B$ band is higher by a factor of $\sim 1.7$. Part of the difference can be due to the fact that L1642 is seen towards a slightly lower Galactic latitude, but our simulations show that this effect explains only a difference of $\sim 10 \%$ in brightness. Because L1642 and IRC +10216 are located nearly symmetrically on the opposite sides of the Galactic plane, it can be assumed that the ISRF impinging on the clouds is similar. Therefore, it seems that the dust grains in the envelope of IRC +10216 must have lower $\omega$ or higher $g$ (or both) than those in L1642. Laureijs et al. (1987) find that the surface brightness of L1642 can be explained with the ISRF model from Mattila (1970), which is similar to the models used in this paper, and grain properties $\omega \approx 0.48, g \approx 0.75$. Observations of other high latitude dark clouds, e.g. in Mattila (1979), also suggest that the surface brightness of IRC +10216 is exceptionally low. Thus, it seems likely that the difference between the observed and predicted brightness of IRC +10216 is caused by inappropriate dust parameters rather than by the ISRF being grossly different from the models used in this paper.

Simulations of cloud colours show that with the dust models employed here the ISRF of Porter \& Strong (2005) predicts too blue $U-B$. Only for grain sizes $a=0.15-0.25 \mu \mathrm{m}$ the simulated $U-B$ is barely within the errorbars of the observations, but the predicted $B-V$ is then much too red. With the Mattila (1980) ISRF the simulated colours fit the observations much better and grain sizes $a=0.06-0.12 \mu \mathrm{m}$ predict colours matching the measurements. For grain sizes $a \gtrsim 0.2 \mu \mathrm{m}$ indicated by the shape and the maximum brightness of the cloud, $B-V$ is $0.3-0.5$ mag too red. It is possible that, e.g., adding a population of small grains could improve the fit of colours, but we have not pursued this line of study further. Given that also the discrepancy between the observed and simulated $B$ band intensities suggests that the dust model needs to be improved, it is premature to draw conclusions regarding the ISRF colours.

If the ISRF intensity is known, it is possible to determine the albedo and the scattering asymmetry parameter directly from the observations. The observed peak surface brightness constrains the dust model to a curve in the $(g, \omega)$-plane (cf. Fig. 8). The observed core depth determines another curve in the plane, and the intersection of the curves yields the parameters of the dust model. The peak brightness and the core depth are not very sensitive to small changes in the cloud density structure, and therefore the determination of the grain scattering parameters is quite robust. Similar methods for constraining the dust properties were used in Mattila (1970) and Fitzgerald et al. (1976). In the present case, however, the uncertainty in true core depth does not warrant using the method for determining the dust properties.

The observed small size of the nebula, i.e. its faintness at large offsets $\left(>25^{\prime \prime}\right)$, compared to simulation results was noted in MLL03. They were unable to produce simultaneously the small size and the correct shape of the profile. With small mass-loss required by the small size, their simulations resulted in profiles with no central depression. In our simulations the central depression is always present, and the small extent of the cloud is easily produced. This difference is not due to our anisotropic incident radiation field, because the same profile shape was observed also in our tests with an isotropic ISRF. Indeed, while in MLL03 it was found that a relatively high radial optical depth (i.e. optical thickness from the centre of the cloud to the outer edge) was needed to produce a central depression, in our simulations it is seen already with radial optical depth of $\sim 2^{2}$. The development of the dark core at fairly low radial optical depths was already observed in Mattila (1970) and Witt \& Stephens (1974). In MLL03 it was also noted that they were unable reproduce the results of MR87 using the same cloud and dust models. In our tests the results agreed with those shown in MR87.

Lack of tight constraints for the dust grain size and the poorly known cloud surface brightness at large offsets hinders the determination of the cloud density structure. Nevertheless, it can be concluded that there are clear signs of the mass-loss rate increasing with time, i.e. $\dot{M}_{\mathrm{d}}>0$. A $r^{-2}$ density distribution corresponding to a constant mass-loss rate does not fit the observations. With the exponentially changing mass-loss used, the best-fit rate constant $\alpha$ varies very little with grain radius, but without more accurate knowledge of the brightness of the outer parts only a rough estimate $\alpha=(3 \pm 1.25) \times 10^{-4} \mathrm{yr}^{-1}$ can be given. On the other hand, the best-fit value of $\dot{M}_{\mathrm{d}}$ changes by a factor of more than 10 within the range of particle sizes deemed acceptable based on the lack of shape chromatism. We emphasize that the mass-loss history is probed using only the outer part of the envelope ( $>12^{\prime \prime}$, corresponding to mass loss more than 500 years ago), and should not be considered as accurately representing the recent mass-loss history. Even in the case of determining the mass-loss rate in the more distant past, the results depend on the chosen model family of exponentially changing mass-loss.

The density structure of the outer envelope of IRC +10216 was analysed by Tamura et al. (1988) using polarimetric observations in $H$ and $K$ bands. They concluded that the distribution of polarized intensity is consistent with an inverse-square dust density distribution between offsets $15^{\prime \prime}$ and $60^{\prime \prime}$, implying steady mass-loss. From fitting a power-law density, $\rho \propto r^{-c}$, to the data Tamura et al. (1988) found $c=2.1 \pm 0.3$ from the $K$ band data, and $c=2.4 \pm 0.3$ from the $H$ band data; thus, their results actually seem to indicate a density law slightly steeper than $r^{-2}$. Comparing their results with our calculations, it can be seen that the inferred density structures are in fact quite similar. Choosing, e.g., $c=2.3$ one gets from a power-law density $\rho\left(15^{\prime \prime}\right) / \rho\left(60^{\prime \prime}\right) \approx 24.3$, while with $\alpha=2.2 \times 10^{-4} \mathrm{yr}^{-1}$ our

2 In MLL03 it was reported that a radial optical depth of $\gtrsim 80$ is needed to produce a dark core. However, their cloud model had a smaller inner radius of the cloud than ours and therefore with the same mass-loss rate their clouds have a radial optical depth larger by a factor of $\sim 5$. 
model gives $\rho\left(15^{\prime \prime}\right) / \rho\left(60^{\prime \prime}\right) \approx 24.2$. In Tamura et al. (1988) the density structure was obtained using a relatively simple model that assumed an optically thin envelope and isotropically scattering dust grains. These assumptions might not be valid at near infrared wavelengths. Detailed simulations of the polarized intensity could be very useful in constraining the dust properties and the cloud density distribution.

Simulations with shell structures similar to what is observed in IRC +10216 indicate that the global effect of the shells is small, and results from simulations with smooth, spherically symmetric clouds are a good approximation. This is in contrast to the case where the surface brightness is due to the scattered light from the central star. Our simulations with parameters suggested for the dust shells in Mauron \& Huggins (2000) produce images resembling actual images of the envelope surrounding IRC +10216 . We have not, however, explored the effect of changing the shell parameters, and therefore we cannot conclude how robust the estimates of their values are. A detailed study of individual shell brightness profiles, measured accurately by Leão et al. (2006), would be an interesting future study in this direction.

More detailed simulations, especially exploring the effect of inhomogenous and aspherical grains and more detailed size distributions is necessary to reach firmer conclusions about the properties of the dust envelope of IRC +10216 and the incident ISRF. In addition to simulations, some more observational data would be useful in constraining the model parameters. Brightness profiles obtained from deeper images of the outer parts could be used to explore the mass-loss history in much more detail and in the more distant past. On the other hand, a more accurate determination of the depth of the dark core in $U$ and $B$ bands is needed to study the asymmetry parameter of the scattering function. Also, further observations of the surface brightness at both shorter and longer wavelengths could be used to study the shape chromatism and therewith to constrain the dust model.

\section{Conclusions}

(1) The absence of shape chromatism indicates that the dominant grain size must be large. The lower limit for grain size is found to be $0.1 \mu \mathrm{m}$. The relatively shallow central depression sets a tentative upper limit of $0.4 \mu \mathrm{m}$.

(2) The shape of the brightness profiles clearly shows that the cloud density structure is steeper than $r^{-2}$, suggesting increasing mass-loss rate with time. The shape of the observed brightness profiles can be reproduced very well with an exponentially changing mass loss that increases 20-50\% in 1000 years. More accurate determination of mass-loss history is not possible due to the poorly known surface brightness of the outer parts of the envelope. Uncertain dust properties, particularly the lack of a tight upper limit for the grain size, prevent us from determining the absolute value of dust mass-loss with good accuracy.

(3) Dust models giving acceptable fits to cloud shape at different wavelengths all produce higher than measured peak brightness, if the ISRF intensities resulting from the Galaxy models of Mattila (1980) or Porter \& Strong (2005) are used. With grain sizes $0.1 \lesssim a \lesssim 0.2 \mu \mathrm{m}$ the discrepancy in peak brightness in the $B$ band is more than a factor of 2 , but grains with $a>0.25 \mu \mathrm{m}$ all give an almost identical difference of $\sim 35 \%$. There is also a mismatch in colours for all grain sizes $a \gtrsim$ $0.12 \mu \mathrm{m}$ with Mattila's ISRF, and for the ISRF of Porter and Strong no grain size can reproduce the observed colours.

(4) The differences between the observations and our simulation results notwithstanding, we consider it premature to conclude that the ISRF models are inappropriate. It is probable that at least part of the discrepancy is due to the dust grains in the IRC +10216 envelope having different scattering properties from our dust models, in particular lower albedo. Simulations with more detailed dust models, including non-spherical grains, are needed to study this issue.

Acknowledgements. We thank K. Mattila for his comments on the manuscript and the referee, N. Mauron, for his useful remarks. We acknowledge the financial support of the Academy of Finland grant 107701.

\section{References}

Bagnulo, S., Doyle, J. G., \& Griffin, I. P. 1995, A\&A, 301, 501

Crabtree, D. R., McLaren, R. A., \& Christian, C. A. 1987, in Late Stages of Stellar Evolution, ed. S. Kwok, \& S. R. Pottasch, ASSL, 132, 145

Crabtree, D. R., \& Rogers, C. 1993, in Mass Loss on the AGB and Beyond, ed. H. E. Schwarz, 255

Fitzgerald, M. P., Stephens, T. C., \& Witt, A. N. 1976, ApJ, 208, 709

Groenewegen, M. A. T. 1997, A\&A, 317, 503

Groenewegen, M. A. T., van der Veen, W. E. C. J., \& Matthews, H. E. 1998, A\&A, 338, 491

Henyey, L. G., \& Greenstein, J. L. 1941, ApJ, 93, 70

Juvela, M. 2005, A\&A, 440, 531

Juvela, M., \& Padoan, P. 2003, A\&A, 397, 201

Knapp, G. R., \& Morris, M. 1985, ApJ, 292, 640

Laor, A., \& Draine, B. T. 1993, ApJ, 402, 441

Laureijs, R. J., Mattila, K., \& Schnur, G. 1987, A\&A, 184, 269

Leão, I. C., de Laverny, P., Mékarnia, D., de Medeiros, J. R., \& Vandame, B. 2006, A\&A, 455, 187

Leinert, C., Bowyer, S., Haikala, L. K., et al. 1998, A\&AS, 127, 1

Martin, P. G., \& Rogers, C. 1987, ApJ, 322, 374

Mathis, J. S., Mezger, P. G., \& Panagia, N. 1983, A\&A, 128, 212

Mathis, J. S., Whitney, B. A., \& Wood, K. 2002, ApJ, 574, 812

Mattila, K. 1970, A\&A, 9, 53

Mattila, K. 1979, A\&A, 78, 253

Mattila, K. 1980, A\&AS, 39, 53

Mauron, N., \& Huggins, P. J. 1999, A\&A, 349, 203

Mauron, N., \& Huggins, P. J. 2000, A\&A, 359, 707

Mauron, N., de Laverny, P., \& Lopez, B. 2003, A\&A, 401, 985

Men'shchikov, A. B., Balega, Y., Blöcker, T., Osterbart, R., \& Weigelt, G. 2001, A\&A, 368, 497

Olofsson, H., Johansson, L. E. B., Hjalmarson, A., \& Nguyen-Quang-Rieu 1982, A\&A, 107, 128

Pegourie, B. 1988, A\&A, 194, 335

Porter, T. A., \& Strong, A. W. 2005, in Proc. 29th International Cosmic Ray Conference (Pune), 77

Rouleau, F., \& Martin, P. G. 1991, ApJ, 377, 526

Skinner, C. J., Justtanont, K., Tielens, A. G. G. M., et al. 1999, MNRAS, 302, 293

Skinner, C. J., Meixner, M., \& Bobrowsky, M. 1998, MNRAS, 300, L29

Struve, O., \& Elvey, C. T. 1936, ApJ, 83, 162

Struve, O., \& Story, H. 1936, ApJ, 84, 203

Suh, K.-W. 2000, MNRAS, 315, 740

Tamura, M., Hasegawa, T., Ukita, N., et al. 1988, ApJ, 326, L17

Toller, G. N. 1981, Ph.D. Thesis

Tuthill, P. G., Monnier, J. D., Danchi, W. C., \& Lopez, B. 2000, ApJ, 543, 284

Voshchinnikov, N. V., Il'in, V. B., \& Henning, T. 2005, A\&A, 429, 371

Wallerstein, G., \& Knapp, G. R. 1998, ARA\&A, 36, 369

Weingartner, J. C., \& Draine, B. T. 2001, ApJ, 548, 296

Witt, A. N., \& Stephens, T. C. 1974, AJ, 79, 948

Witt, A. N., Oliveri, M. V., \& Schild, R. E. 1990, AJ, 99, 888

Young, K., Phillips, T. G., \& Knapp, G. R. 1993, ApJS, 86, 517 